\title{
Petits arrangements avec le développement durable
}

Entre production scientifique et instrumentalisation médiatique

Véronique André-Lamat, Laurent Couderchet et André-Frédéric Hoyaux

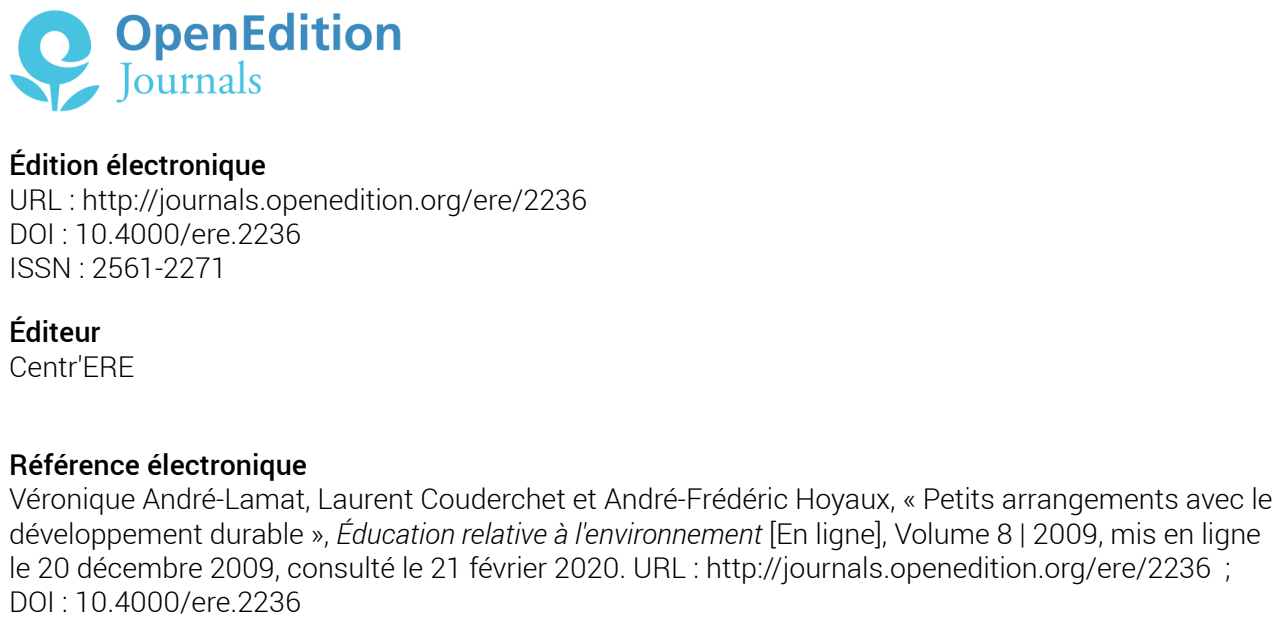

Ce document a été généré automatiquement le 21 février 2020. 


\title{
Petits arrangements avec le développement durable
}

\author{
Entre production scientifique et instrumentalisation médiatique
}

Véronique André-Lamat, Laurent Couderchet et André-Frédéric Hoyaux

1 Dans l'univers de la communication, l'enjeu environnemental, qui s'opposait jadis au productivisme économique, structure désormais l'ensemble des discours et des stratégies. Les industriels, pointés du doigt par les écologistes, transforment les contraintes environnementales en potentiels commerciaux ou les intègrent dans leurs systèmes de décisions opérationnels : en amont, à travers les comités d'éthique ou les "clubs de développement durable», voire les fondations d'entreprises; en aval, à travers la mise en place de normes maquillées en externalités positives pour l'humanité. La question environnementale, devenue populaire et incontournable tandis que s'affirmait l'idée d'un développement durable, est ainsi devenue un produit d'appel, une ressource en elle-même. Les collectivités territoriales et les associations participent également de cette mobilisation, de cette convocation à l'environnement, qui s'organise suivant différentes modalités et différentes approches.

2 À l'occasion des travaux français du Grenelle de l'environnement décentralisé à Périgueux, la question de l'éthique en éducation à l'environnement a notamment porté sur le «marketing écologique», seul sujet sur lequel les participants de l'atelier consacré à la "démocratie écologique " ont envisagé de proposer un recours à la réglementation. Les partenaires réunis à cette occasion ont beaucoup insisté sur la nécessité d'un renforcement de l'éducation à l'environnement ou au développement durable, destiné à améliorer les capacités d'appréhension de problématiques complexes et incertaines pour une implication citoyenne éclairée dans le débat territorial participatif. L'article 8 de la Charte de l'environnement récemment intégré en préambule de la Constitution française au même niveau que la Déclaration des droits de l'Homme de 1989 ne précise-t-il pas que : «L'éducation et la formation à l'environnement doivent contribuer à l'exercice des droits et devoirs définis par la présente Charte »?

Dans cet article, nous proposons d'interroger et d'analyser la question du marketing écologique, en nous appuyant sur un corpus documentaire composé de slogans, 
d'iconographies, mais aussi d'extraits de sites Web ou de discours' ${ }^{1}$. Ce point de départ amène ainsi à confronter éthique de l'environnement, éthique de la communication et in fine éthique de l'éducation.

L'éthique vise à examiner des questions et essayer de comprendre leurs implications, plus qu'à donner des solutions (Dolan, 1999). Ainsi, selon la circulaire $n^{\circ}$ 2004-110 du 8 juin 2004 de l'Éducation nationale relative à la "généralisation d'une éducation à l'environnement pour un développement durable ", l'éducation à l'environnement et au développement durable renvoie à cette dimension éthique. Il s'agit tout d'abord de mobiliser l'approche systémique pour appréhender et faire comprendre la complexité des phénomènes et des réponses à apporter aux problèmes environnementaux. Il s'agit également de développer une approche critique, de mettre « en valeur l'importance des choix et la responsabilité de chacun dans ses choix », d'intégrer " les valeurs associées à un développement solidaire ». En cela, l'éthique participerait d'un tournant herméneutique au sens où il n'y aurait plus d'interprétation «scientifique » univoque (une objectivité) réalisée par un interprétant unique (le scientifique qui objective), mais un ensemble de rapports entre constructions multiples du sens sur un même objet (interobjectivité) et une multitude d'interprétants plus ou moins constitués en collectifs sociaux (associations) ou professionnels (scientifiques).

5 Si l'on part du postulat qu'éduquer, c'est (en partie) apprendre à comprendre des modèles et tout autant apprendre à les construire, alors une éducation efficace suppose certes une transmission de connaissances, mais aussi de valeurs qui s'établissent en référence aux modèles. Or l'éducation doit construire la capacité à critiquer: « Si jamais vous substituez dans son esprit l'autorité à la raison, il ne raisonnera plus ; il ne sera plus que le jouet de l'opinion des autres " (Émile ou De l'Éducation - Rousseau, repris par Philippe Meirieu, 2007). En ce sens, un des défis de l'éducation est bien d'apprendre à déconstruire des modèles, à décoder les discours qui les sous-tendent, à gérer l'incertitude et acquérir la capacité de construire individuellement une responsabilité, un engagement envers un projet collectif pour un bien commun. Alors, "éduquer à l'environnement " puis "éduquer au développement durable $»^{2}$, renvoie en partie à l'éthique de l'environnement ${ }^{3}$, qui préconise de la retenue dans l'exploitation de l'écosphère. De la même manière, dans le discours construit sur l'environnement, la démarche éthique relève certainement d'une retenue, tant les problématiques en cause ont parfois des fondements incertains. Dans sa démonstration d'un glissement vers la pensée unique, Georges Rossi (2000) fustige une pensée écologique qui «sous couvert de justifications morales et scientifiques, transforme l'hypothèse en vérité, la probabilité en certitude et en idée reçue ».

6 Au-delà de l'objectif de construire un modèle (éthique ?) de compréhension de l'environnement, voire d'approches et d'usages éthiques de l'environnement, l'éducation au développement durable a une double vocation. Elle vise tout d'abord à améliorer les capacités d'appréhension de problématiques complexes et incertaines. Le passage de l'environnement au développement durable a rétabli institutionnellement les interactions entre le système écologique et son environnement (au sens systémique) et il a réintégré les acteurs économiques dans les problématiques environnementales. Le degré de complexité à appréhender a été fortement accru par la notion de développement durable, notamment parce qu'elle impose de prendre en compte plusieurs interprétants de cette complexité. Elle introduit ensuite explicitement un précepte nouveau, celui de l'implication citoyenne dans les projets, et fait émerger un 
besoin indispensable d'éducation. Cette éducation se déroule en formation continue. Aux plus jeunes, elle est dispensée en principe par l'éducation nationale qui propose des programmes et des instructions de plus en plus précis pour réduire la part d'interprétation qui pourrait naître de la diversité des intervenants. Aux plus grands, la formation passe par la compilation d'un ensemble d'informations produites par une palette médiatique de plus en plus conséquente. Pour autant, la chaine d'information est très souvent circulaire et propose des liens qui se renvoient les uns aux autres: entre les types de médias (Internet reprenant des informations des journaux télévisés reprenant des informations de quotidiens, par exemple) et à l'intérieur même d'un type de média (des sites Internet reprenant des informations d'autres sites Internet). Ces renvois mutuels créent une sorte de convergence sémantique qui freine la construction d'un esprit critique.

Or, une vraie participation nécessite un minimum de connaissances (ou d'expériences). En ce sens, l'éducation au développement durable devrait aussi s'appliquer à forger une implication citoyenne "éclairée» dans le débat territorial participatif, ce qui interroge: la participation doit-elle seulement intervenir a posteriori, dans l'action territoriale ? Ou doit-elle s'envisager dans le cadre même du projet d'éducation?

8 L'éducation à l'environnement et, ou au développement durable ne concerne donc pas seulement les enfants, collégiens ou lycéens ; elle s'adresse aussi aux adultes citoyens et renvoie à une certaine conception du civisme. Une des questions clés est alors : qui participe à construire cette éducation et les modèles qui y sont associés ? Est-ce l'école ou l'université ? Est-ce les associations de défense ou d'éducation à l'environnement? Ou est-ce la télévision et la publicité qui propose des modèles dont la légende implicite ou explicitée génère dans un même package, discours et interprétation du discours ?

$9 \mathrm{Au}$ regard de l'idée que le spectaculaire (Debord, 1996) est l'instrument de médiatisation des enjeux environnementaux et que de nombreux agents et modes de communication s'en sont emparés, la question de l'éthique en éducation à l'environnement porte en particulier sur le «marketing écologique». Lorsque le marketing écologique devient vecteur d'éducation (ou de pédagogie?), que s'instaure via ce marketing une relation dominant/dominé (producteur du message/récepteur du message), que des enjeux économiques ou idéologiques se manifestent, alors le message se brouille au gré des intérêts des producteurs du message.

Notre analyse sémiotique de l'image de publicités et de discours (Sauvageot, 1987 ; Joly, 1993; Gervereau, 2004) fait le constat d'une production scientifique happée par des outils de communication qui conforment les regards sur le développement durable.

11 Deux catégories de slogans (d'approches?) sont retenues ici : celle qui annonce la victoire de l'inventivité de l'industrie sur la crise écologique, celle qui recycle et réorientent les analyses et les points de vue sur les grandes questions scientifiques pour minorer, simplifier ou détourner la réalité.

\section{Justifier le modèle social et économique, un certain regard sur le développement durable}

12 La définition du développement durable proposée en 1987 par le rapport Brundtland est consensuelle, l'ensemble des catégories d'acteurs (politiques, économiques, associatifs, etc.) s'en est emparé. Ce consensus «mou» s'explique par les multiples 
interprétations qu'elle autorise. En simplifiant et sans rentrer dans les nombreux débats sur le sujet, des approches plus "économicistes" et des approches plus " écologiques » et normatives se distinguent ${ }^{4}$.

L'engouement pour l'environnement des grandes entreprises surfant sur la vague du développement durable se traduit dans les stratégies de communication. Le vocabulaire et l'iconographie renvoient à une nouvelle rhétorique, mobilisent de manière constante les mots " nature ", « environnement ", « équité », « responsabilité », « éthique ». Leur récurrence dans les discours a pris une telle ampleur que dès 1998 le Bureau de vérification de la publicité (BVP, de l'Association des professionnels pour une publicité responsable) puis le Conseil d'éthique publicitaire se penchent sur la question. Le BVP édite des recommandations sur l'argumentaire écologique, puis en 2007 sur l'argumentaire développement durable. En partenariat avec l'ADEME (Agence de l'environnement et de la maîtrise de l'énergie), un observatoire est monté et un rapport publié : La publicité est-elle respectueuse du développement durable? (ADEME et BVP, 2007). La teneur du message véhiculé par le biais des slogans, des mises en scène et de l'iconographie est bien aujourd'hui le cœur d'une réflexion qui touche à la capacité de recevoir, décoder, interpréter un message publicitaire. Ce message est par nature simplificateur, mais dans quelle mesure peut-il s'avérer manipulateur, peut-il être instrumentalisé, peut-il générer voire justifier des comportements "non éthiques ", dès lors qu'il participe (consciemment ou non) à construire une certaine vision des problèmes environnementaux et $\mathrm{du}$ " comment faire du développement durable »?

Sur un marché devenu très concurrentiel, les entreprises considèrent manifestement que les thématiques de l'environnement et du développement durable constituent non seulement une opportunité commerciale, mais aussi un facteur positif de leur image. Deux exemples sont ici analysés concernant les multinationales Véolia et Total, plutôt considérées comme de bons élèves par le BVP.

\section{« L'environnement est un défi industriel »}

16 Véolia nous embarque dans le champ de l'environnement par la mobilisation d'une figure emblématique des problèmes environnementaux qui a fait ses preuves : l'arbre. L'arbre renvoie aux discours médiatisés sur la déforestation, l'Amazonie, le " poumon vert » de la planète. C'est un symbole immédiatement identifiable pour caractériser les enjeux environnementaux. Considérant qu'elle correspond à «la prise en compte des multiples liens sensibles à l'environnement " (Blanc, 2008), l'esthétique de l'image est irréprochable. Son organisation et la dominante de vert participent à produire une composition imaginaire d'un lieu qui a un sens écologique pour qui le contemple.

17 La figure de l'arbre est parfaite avec des axes de communication routiers constituant le réseau de branches, de nervures qui l'irrigue et le nourrit: message dont la clarté environnementale peut paraître paradoxale. Mais l'analogie est cohérente au regard du petit paragraphe explicatif. En effet, aucune remise en cause de l'augmentation de la consommation d'eau et d'énergie, de l'augmentation de la production de déchets, etc. n'est envisagée. Pourquoi le seraient-elles puisque ces fameuses nervures ne sont que les représentations des «flux » nécessaires qui irriguent les villes (comme la sève pour l'arbre) et qui sont gérés avec tant de «naturel » par Véolia! Un simple constat donc de "l'indispensable» nécessité de ces déplacements d'énergie, auquel il faut « simplement » trouver une solution grâce à une gestion efficace. 
18 «L'environnement est un défi industriel». Le slogan de Véolia réduit ainsi l'environnement à des solutions dont dispose l'entreprise grâce à des techniques et des métiers, et donne peut-être raison à ceux qui militent pour le maintien de l'expression « éducation relative à l'environnement » (Sauvé, 2002). Au-delà, il y a implicitement un positionnement singularisant, Véolia est l'arbre qui cache la forêt (les autres entreprises ?).

\section{L'environnement un bien économique à cultiver}

19 Comme celui de Véolia, le message de Total vise à rassurer, et même à démobiliser le citoyen consommateur: ne changez pas de mode de consommation, Total assure et assurera l'approvisionnement. "Pour vous, notre énergie est inépuisable ».

Le parallèle entre la débauche d'électricité et le jaune infini d'un pseudo champ de colza est à ce titre édifiant. Les images se répondent, s'appuient sur la couleur chaude et réconfortante et participent par l'esthétique à construire finalement «une représentation de l'espace sensible dans lequel nous vivons et auquel nous aspirons » (Blanc, 2008). La stratégie n'est pas de nier, mais de contourner le problème de l'énergie, de l'inscrire dans un paradigme qui ne remette pas en cause le modèle de développement économique. L'enjeu pour Total est clairement économique : il s'agit de "répondre à la demande». La référence aux carburants du futur renvoie à une utilisation de la nature qui peut paraître antinomique avec des objectifs de préservation de l'environnement, de limitation d'exploitation des ressources. Total entretient une vision du développement durable qui réconcilie la consommation et la nature, en proposant de remplacer des biens et services non renouvelables par des formes renouvelables (biocarburants et agrocarburants sans distinguo) : « pour mettre au point les carburants, nous faisons appel à la nature ». L'insecte butineur présent sur une fleur au premier plan est destiné à rassurer sur le caractère environnemental de l'agriculture fournisseur des carburants du futur. Total ne promeut-il pas la biodiversité à travers sa fondation? Ici le positionnement est double, il s'agit de montrer : premièrement, à travers l'image de l'abeille, le changement des manières de faire, la substitution progressive d'une externalité négative (symbolisée par l'image du haut, une usine qui dans l'imaginaire pollue, fait du bruit) par une externalité positive (symbolisée par l'image du bas, cette abeille qui butine et dont la portée symbolique relève de la nature auto-productive, donc paradisiaque puisque l'Homme n'y a pas à faire d'efforts) ; deuxièmement, au-delà de cette substitution somme toute utopique, la nécessité de mettre en place quelque part des technologies pour transformer et amener cette énergie auprès des Hommes.

21 Enfin la publicité montre aussi la capacité des Hommes à faire du beau à travers ce déferlement technologique. Reprenant le titre du livre de Richard Bohringer et le modifiant quelque peu, comment ne pas se dire en voyant l'image que «c'est beau une usine la nuit »!

22 Comme dans les spots radio et de télévision de tous les fournisseurs d'énergie, le message est assorti de la formule pudique réglementaire : "l'énergie est notre avenir, économisons-la». À l'instar des formules «fumer tue» et "mangez au moins cinq fruits et légumes par jour » imposées aux marques de tabac et à l'alimentation, la puissance publique impose une formule dérisoire censée contribuer à la conscientisation des masses ${ }^{5}$. Sur l'affiche, la mention obligatoire écrite en caractères 
ridiculement petits est accolée à un slogan ambigu et contradictoire du message officiel, beaucoup plus visible celui-là: "notre énergie est inépuisable». La multinationale qui se pose en organisation responsable («carburants et produits respectueux de l'environnement »), pense le futur pour nous. Elle adopte une double stratégie: de culpabilisation et de sécurisation (inquiéter pour mieux sécuriser), l'objectif n'est pas de négation, mais de contournement.

Plus globalement, Total a pris conscience de la nécessité de ne pas faire peur aux consommateurs sur l'éventualité qu'ils n'aient plus accès "facilement ", c'est-à-dire en tout lieu et à tout moment, aux services énergétiques. Pour ce faire, l'idée est de forcer le trait et de se positionner sur une symbolique ubiquiste (être partout en même temps) et uchroniciste (être en tout lieu sans qu'il n'y ait besoin de faire des efforts par rapport à une chronologie particulière : exploiter un gisement, transporter la matière première, travailler cette matière première jusqu'aux consommateurs). Ces spécificités qui hier encore étaient l'apanage de Dieu semblent donc maintenant partagées.

Comme la publicité Véolia, la publicité Total envoie, toujours dans un esprit de sécurisation, un message subliminal au consommateur. Sur la première image, un bosquet dessine la silhouette d'une girafe, sur la seconde, les fleurs sont butinées par une abeille surdimensionnée, ce qui doit nous rassurer sur les pratiques culturales à l'origine de la matière première. L'analyse de ces deux exemples nous amène à constater la production d'un discours aussi flottant que la notion même de développement durable, qui s'appuie finalement sur la nature productive (Total), l'environnement submergé, mais reconstruit par les productions humaines (Véolia). Il n'est cependant aucunement question de réviser un mode de consommation, un mode de développement, mais plutôt de le conforter. On aurait d'ailleurs pu mobiliser de la même manière, pour étayer notre propos, une série de publicités sur les véhicules hybrides, produisant moins de $\mathrm{CO}_{2}$, Toyota ou Renault par exemple. Il s'agit clairement de préserver la capacité des sociétés à produire et non pas à conserver telle ou telle ressource. L'objectif est de remplacer des biens et services non renouvelables par des formes renouvelables, ce qui implique une confiance totale dans le progrès technique. La foi en la science et la technologie est ici structurante et conduit l'individu loin de toute évocation d'une crise quelconque de la modernité. La conception et la configuration des techniques organisent la société en la subordonnant à un certain ordre (Feenberg, 2004). On peut enfin se demander s'il n'y a une certaine forme d'ironie machiavélique dans ces publicités à l'adresse de la société: dites-nous vos représentations et vos lubies, nous arriverons toujours à les intégrer dans nos nouvelles technologies!

\section{Interobjectivité et intersubjectivité en question}

Le développement durable, comme l'environnement, a une dimension systémique, c'est un des fondements du rapport Brundtland. Parmi les grands préceptes de la systémique définis par Von Berthalanffi ([1968], 2002), la complexité est associée à l'incertitude. La méthode systémique justifie la simplification dans un but de compréhension des structures et dynamiques des systèmes, cette simplification produisant l'incertitude (Le Moigne, [1977], 2004). Lorsque "l'animation environnementale » devient communication, ce principe simplificateur devient slogan et l'hypothèse se fait conclusion. 
En choisissant de communiquer sur l'environnement, l'univers de la publicité mobilise les grandes problématiques qui intéressent le monde scientifique. Il opère ensuite des simplifications qui génèrent des affirmations. Celles-ci s'appuient sur différentes formes de vulgarisation scientifique qui ont percolé une grande part de la société aujourd'hui, par le biais de personnages emblématiques (non scientifiques) tels que Nicolas Hulot ou Yann Artus Bertrand, nouveaux héros de l'écologie citoyenne, et d'émissions télévisées dites "grand public ». Sans faire une défense de castes, ces personnages sont souvent hors champ des disciplines dont ils prétendent savoir quelque chose. De ce fait, il y a une sorte de connivence avec le public qui arrive alors à travers eux à se projeter lui aussi comme possible commentateur de l'actualité de la crise environnementale. Ces personnages présentent les mêmes symptômes que les nouveaux philosophes sur lesquels Pierre Bourdieu avait travaillé quand ces derniers avaient les faveurs des médias. Aujourd'hui, ces faveurs se tournent vers quelques "scientifiques" semblant avoir du "crédit», mais qui surtout apportent avec eux l'audimat, ce champ économique "très hétéronome » et donc "très fortement soumis aux contraintes commerciales" (Bourdieu, 1996, p. 62). Cette hétéronomie était traduite par Bourdieu comme la possibilité donnée à quelqu'un qui n'est pas spécialiste dans un champ de recherche de faire des commentaires en tant que spécialiste dans ce champ ou de pouvoir juger un spécialiste de ce champ comme s'il l'était lui-même.

Un jeu d'autorité se construit donc entre journaliste, spécialiste supposé et spécialiste tout court. Et la partie pédagogique n'est évidemment pas gagnée quand celle-ci s'inscrit dans le jeu du classement qui relève plus de l'affectif que de l'effectif! Hier, Bourdieu relevait que «les hebdomadaires adorent ça: faire le bilan de la décennie, désigner les dix grands «intellectuels» de la décennie » (Bourdieu, 1996, p. 66-67). Aujourd'hui, l'Express dans un numéro spécial «Agissons maintenant. Urgence écologique » nous propose d'élire nos " héros écolos » : «Connus - ou non - du grand public, ces hommes et ces femmes se battent pour sauver la planète, chacun à sa manière. Que pensez-vous de leur action? » Et de nous permettre ainsi au-delà des six stars en photos (Gore, Hulot, Bertrand, Vanier, Étienne et Di Caprio) de voter pour notre héros: "Vous voulez vous aussi proposer un nom? Envoyez-nous votre proposition par e-mail en cliquant ici [...] $»^{6}$. Derrière ces images de héros écolos se glisse la personnalisation des héros légendaires qui rappelle les grands sauveurs de l'humanité et les histoires plus ou moins vraies, plus ou moins imaginées que nous racontaient au coin du feu nos grands-mères dans leur bonne sagesse... Histoires qui avaient toutes une morale, structurante et structurée depuis des siècles, évoquant en cela la durabilité du bon sens. Pour autant, nous verrons qu'il ne s'agit pas d'opérer une simplification visant à mieux faire comprendre la complexité, mais plutôt d'alimenter la construction de récits, susceptibles de nourrir l'imaginaire et les référentiels collectifs, de fonder de nouveaux mythes. Pour cela, et suivant les conceptions de l'environnement et de l'écologie parfois assez radicale de la Deep Ecology, la planète sera assimilée à un « être vivant ». Cette fois, c'est la Terre qui se trouve personnalisée pour nous la rendre plus chaleureuse, plus proche, comme c'est le cas pour le Gaïa de James Lovelock, ou pour la lecture qu'en fait Jonas dans sa conception ontologique. Autant de systèmes de valeurs qui nourrissent de nouvelles orientations idéologiques et mentales pour les sociétés sur lesquels se positionnent les industriels. 


\section{Éviter la reproduction de « l'écocide » de l'île de Pâques}

28 étant proposées dans des temps différents. Mais au-delà, du fait que les processus à l'œuvre sont plus complexes, il est difficile de les comprendre sans rentrer dans l'explicitation de la méthode utilisée et de ses conséquences sur les résultats proposés. L'action publicitaire ne peut reprendre quelque chose qui relèverait des choix du chercheur et in fine engagerait celui... du consommateur. EDF est le « leader européen des énergies de demain ", mais pour que ce demain soit, il faut faire le bon choix aujourd'hui (celui d'EDF ?) ${ }^{7}$, d'autant que les Hommes doivent bien avoir conscience que comme les habitants de l'île de Pâques piégés sur leur île, ils sont tous piégés sur la Terre. La publicité nous rappelle d'ailleurs « on ne peut pas la quitter ». La finitude irrémédiable de la planète doit donc les inquiéter au regard de leurs choix. Et on peut faire avec le géographe britannique Denis Cosgrove (1994), le rapprochement entre la conception durabiliste actuelle de notre planète et la vue de cette dernière par les astronautes de la NASA posant le premier pas sur la Lune en 1969. Car, voir la terre en perspective, à une très petite échelle, c'est la voir dans son unicité organique indépassable.

31 Cette publicité d'EDF renvoie d'une part au conservationnisme et aux théories néomalthusiennes qui caractérisent certaines conceptions de l'environnement; d'autre part aux problèmes environnementaux les plus «populaires » (déforestation, 
surexploitation des ressources par des sociétés du Sud prédatrices de leur environnement). En revanche, dans notre société moderne de la connaissance et suivant le message d'EDF, miser sur l'exploitation de l'électricité permet de réguler plus rationnellement l'exploitation des ressources terrestres, d'assurer un développement durable et donc de garantir le maintien de la vie sur terre. EDF communique beaucoup sur la recherche, sans jamais évoquer la question du nucléaire, encore moins celle du recyclage des déchets radioactifs. Un tour de force qu'effectue également Areva en parlant du nucléaire comme d'une " énergie au sens propre ». De toutes les entreprises, elle est sans doute la plus aguerrie aux controverses environnementales. Dès le début des années 1970 en effet, l'électricien a dû intégrer à ses stratégies des contestations anti-nucléaires extrêmement virulentes. Sa grande expérience transparaît dans l'efficacité de son message publicitaire.

\section{« Un bébé naît, Guigoz plante un arbre »}

Cette entreprise joue d'une certaine manière sur le même registre de la déforestation et de la démographie, mais aborde la question des naissances de façon originale. Il s'agit tout de même du fondement de sa prospérité...

Le constat de la déforestation est ici finalement un préalable acquis, et Guigoz propose une forme de compensation, destinée à maintenir l'équilibre entre croissance démographique et ressource végétale: " 1 bébé, 1 arbre». On peut également interpréter cette publicité dans une posture métonymique. En effet, on a ici un jeu de similitudes provoquées par la proximité enfant-arbre compris comme les dernières ressources de l'humanité dans son ensemble. La partie, l'arbre, mais aussi le bébé, doit être pensée pour le tout, la forêt et au-delà l'environnement, et au-delà l'ensemble des personnes qui vivent sur notre planète. Donc nourrir un enfant, c'est sauver un arbre, et donc notre environnement, mais inversement, sauver un arbre, c'est aussi nourrir un enfant et notre planète. L'idée de transmission aux générations futures, d'équité intergénérationnelle aussi, inhérente au développement durable, est mobilisée en se fondant sur l'image forte du bébé.

La firme a tout de même le mérite d'associer l'office National des Forêts et nous permet ainsi de penser que le reboisement sera effectué dans les règles de l'art. Ce n'est pas toujours le cas et le geste environnemental peut alors avoir des effets anti-écologiques. Dans un article intitulé "Tempête sur Versailles », Véronique Dassié (2002) explique que la tempête qui balaya la France en 1999 semble avoir changé le statut des arbres: "longtemps destinés à produire de la matière première, ils deviennent dans les discours, plus que des arbres, des êtres vivants qu'il faut protéger et conserver ». Le processus produit par l'événement de 1999 est compassionnel. L'émotion "qui s'enracine dans l'arbre est source d'un élan patrimonial " (Dassié, 2002), cet élan est irrationnel. Plus récemment, dans l'hebdomadaire l'Express (Olivier Le Naire, 2007), Laurent Choffé, adjoint à l'architecte en chef des Monuments Historiques chargé de la restauration du parc de Versailles, confesse qu'à sa manière, cette tempête fut une aubaine «car le public n'aurait jamais admis une campagne d'abattage radicale. Or ces abattages étaient indispensables, puisque les plantations des jardins de Le Nôtre étaient conçues pour être renouvelées tous les cent ans. Pour la plupart, elles en avaient 120. Il était temps d'agir». Nombreuses sont les situations où une pensée écologique 
sommaire des riverains s'oppose désormais à la science des forestiers et empêche des abattages sanitaires d'arbres.

La traduction simpliste de la relation entre l'environnement et l'arbre est en effet récurrente dans le message écologisant. À l'occasion de la promotion de son récent ouvrage C'est bon pour la planète, Evelyne Dhéliat prétendait acheter des sapins de Noël en motte et les replanter par la suite. On ne peut demander à la présentatrice de la météorologie sur TF1 de connaître les programmes de restauration de la qualité écologique des milieux dans le Morvan, derrière les cultures de sapins de Noël ${ }^{8}$. On ne peut lui demander non plus de plonger dans les règlements d'urbanisme qui interdisent, au nom du paysage et du cadre de vie justement, les plantations d'arbres résineux à haute tige dans les quartiers résidentiels. Pourtant, ce type de raisonnement simpliste diffusé à heure de grande écoute, en transformant la cause écologique en niaiserie de consommation courante, peut avoir un effet sur les représentations des populations et donc rejaillir sur le sens de leur implication dans le débat public.

Qu'il s'agisse des slogans qui constituent des raccourcis vertigineux et gomment toute analyse systémique ou d'images chocs, la réduction de la complexité des relations Homme-Nature articule de nombreux discours: ceux des grandes entreprises qui cherchent à valoriser leurs produits et leur image comme ceux des collectivités territoriales ou des associations écologistes. Tous visent un même but, l'efficacité de leur action. Que cette action soit mercantile, politique ou militante.

\section{«Évitons ça!»}

Les pouvoirs publics ne sont pas épargnés par le mouvement qui vise à toucher la corde émotionnelle du citoyen. Il ne s'agit plus là de vendre une énergie soi-disant plus propre, un petit-pot ou un livre, mais de faire la promotion d'une politique publique environnementale. Une campagne de communication du Conseil régional d'Aquitaine montrait récemment une série d'images chocs assorties d'un slogan simple : « évitons ça!».

La première photo rappelle le désastre de décembre 1999, décidément efficace pour susciter l'adhésion, laissant croire que les politiques régionales disposent d'une solution face aux ouragans. Un plan climat régional qui contribuerait à la lutte contre les gaz à effet de serre nous permettrait-il d'éviter de nouvelles tempêtes? Il y a loin, semble-t-il, des moyens d'intervention d'une région française à la maîtrise des fureurs météorologiques. La seconde image montre la sécheresse et suggère un processus d'aridification en marche, à l'image des campagnes de sensibilisation sur la question du Sahel, dans les années 80. Ces campagnes, notamment celles de l'UNICEF, associaient l'argile craquelée par le soleil à la famine et à l'image d'enfants mourant de faim. La dessiccation du lit d'une rivière bien de chez nous doit nous alerter sur l'urgence de la situation en ce qui concerne le réchauffement climatique et la baisse des nappes phréatiques. Cette image doit nous inciter à adhérer aux politiques mises en œuvre par les pouvoirs publics, notamment l'interdiction d'arroser pendant certaines périodes de l'année ou de la journée. Ce phénomène est pourtant récurrent sur les bords vaseux des cours d'eau, il n'est pas forcément catastrophique. C'est bien ici la mise en scène, associée à des référents qui se sont installés dans une culture commune, qui confère à la situation son caractère dramatique. 

l'on pense aux campagnes actuelles du ministère de l'Écologie et de l'aménagement durable pour inciter les citoyens français à éteindre la lumière lorsqu'ils quittent une pièce, ou à fermer le robinet lorsqu'ils se lavent les dents : projet politique ou aveu d'impuissance ? Le discours est normatif, la rhétorique actuelle de la durabilité produit un ensemble de prescriptions qui devraient faire effet dans quelques dizaines d'années, suffisamment longtemps pour que nos technologies aient changé. Ce genre de message peut paraître dérisoire, voire stérile quant à son impact, mais il n'est pas sans " performativité ». Cette dernière est en fait une conception étayée par le philosophe John Austin ([1962], 1991) dans son ouvrage Quand dire, c'est faire. Pour lui, l'action du discours peut parfois être action tout court, même s'il peut y avoir découplage temporel entre les deux. Et ce que nous demandons en tant que citoyens, c'est justement que nos élus agissent. À défaut de faire, les élus peuvent dire et surtout rendre visible cette action de dire. Et quoi de mieux que la publicité pour compléter ces deux axiomes sociologiques et combler le vide politique : action et visibilité de l'action.

\section{La science participe}

Le monde associatif n'est pas épargné par la nécessité de cette communication racoleuse. Sa légitimité est assise sur son désintéressement ${ }^{9}$, elle est parfois confortée par le soutien scientifique. Indépendamment de la justesse du combat de l'association et de son intégrité, le slogan du WWF ("Le Rhône : un désastre écologique ») pose au chercheur un problème éthique : quelle crédibilité accorder aux résultats d'une étude, fut-elle réalisée par 300 médecins, qui déclare ses conclusions avant d'être lancée? L'opération coup de poing joue de notre crédulité : l'eau du Rhône serait en fait devenue un produit irritant ou nocif puisqu'elle est explicitement symbolisée par la croix au milieu de РCB. Croix plus directement compréhensible que le PCB (polychlorobiphényles) lui-même. Ce produit hautement toxique pour l'être humain en général (en bout de la chaîne alimentaire) et pour le milieu halieutique en particulier nous renvoie à l'idée d'empoisonnement qu'évoque la bouteille avec le squelette du poisson. Le slogan «Sommes-nous contaminés?» induit une réponse affirmative. L'idée de cette contamination et de sa découverte par une association qui ne relève pas des pouvoirs publics tend à nous faire croire que l'administration publique dans son ensemble et sanitaire en particulier nous cache quelque chose. On joue ici du ressort d'un imaginaire qui a trait aux légendes urbaines, sorte de rumeurs fondées (ou infondées peu importe) qui traversent les discours de café du commerce, et qui s'étayent de jour en jour, en boucle, pour fonder une intersubjectivité latente qui ne demande qu'à se renforcer lors de nouvelles révélations.

Cette intersubjectivité trouve sa légitimité dans le poids pédagogique de l'autorité scientifique représentée non par WWF, mais par les médecins préleveurs. Ces derniers sont entrés en résistance par rapport à l'administration sanitaire, les « collaborateurs » de l'État. Mais à force de rhétorique, le lecteur se perd dans ses propres capacités d'action. L'accusation existe, mais elle n'a pas d'accusé direct à mettre dans le box, et comme les personnes lésées, le citoyen se retrouve sans comprendre pourquoi il en est ainsi, quel est le mobile, pourquoi et par qui l'acte a été commis. 


\section{Analyse cognitive de l'action}

Dans ces exemples retenus parmi d'autres, les acteurs économiques, politiques et associatifs construisent des mythes ou des récits accessibles à tous. Ils convoquent la science pour vendre ou pour justifier leur a priori, détournent des images pour interpeller les citoyens et ouvrir des fenêtres dans l'agenda politique. Dans cette démarche, il n'est plus question de l'incertitude consubstantielle des problématiques environnementales. Les thématiques accrocheuses de la déforestation, du désert qui avance ou de la surpopulation sont notamment utilisées car elles permettent de mobiliser l'heuristique de la peur. Pour éviter les catastrophes naturelles et humaines irréversibles (comme celle de l'île de Pâques), il faut suivre les préconisations soit des multinationales comme EDF ou Guigoz qui travaillent à notre bien commun, soit des collectivités territoriales qui contribuent à nous assurer un environnement quotidien vivable, soit enfin des associations qui réveillent en nous notre conscience de citoyen du monde responsable, ayant des devoirs envers la planète. Mais si la peur est un moyen historique, universel et sans faille pour orienter la conscience collective, elle renvoie à deux courants philosophiques différents : celui mobilisé ici qui considère la peur comme un outil heuristique et éthique (Jonas); un autre qui considère l'heuristique de la peur comme une manipulation politique terrorisante, susceptible de produire une dérive totalitaire au nom du bien (Huxley, [1932], 1977). Enfin, avec la peur, les publicités évoquées jouent aussi sur le grand classique que constituent le sentiment de culpabilité et la culpabilisation. Culpabilité qui recourt à notre inconscient collectif occidental imprégné des conceptions religieuses dualistes (le bien et le mal, l'enfer et le paradis) qui nous amène à faire un choix, celui de l'action vers le bien. Actions vers le bien qui sont souvent prédéfinies par des autorités hiérarchiques, sortes de tutelles morales plus ou moins manipulatrices et elles-mêmes manipulables. Le Conseil Régional, qui nous amène à ordonner nos consciences par la publicité, ne l'est-il pas par divers lobbies industriels ou associatifs?

Comment dépasser ce type de construction? Comment passer d'une culpabilisation " passive » à une responsabilisation active ? Comment aussi construire quelque chose qui relève plus de l'éducation civique, c'est-à-dire développer les capacités du citoyen à s'investir et produire du projet collectif? En changeant d'échelle peut-être, et en ciblant un niveau d'appréhension de l'espace pour l'individu, mais qui se construit à travers l'action de tous: l'espace de proximité. La réhabilitation du local est bien inscrite dans les orientations du développement durable, au sens de l'espace de vie, de l'espace du quotidien.

Finalement, à travers les différentes conceptions des modèles économiques (croissance ou décroissance), des idées demeurent, il faut instruire la mise en forêt des espaces. Il y a donc bien là un point d'ancrage de l'interobjectivité dans ce qu'elle a de plus normatif et en tant qu'elle se substitue à tout esprit critique !

\section{Pour conclure}

Dans un contexte présenté "d'urgence environnementale ", face à quelques scandales retentissants et à la pression sociétale, les entreprises ont connu une fragilisation de leur image et se sont engagées dans des démarches dites éthiques: "La morale commande, l'éthique recommande» (Comte-Sponville, 1991). Mais l'aspect volontaire 
de ces démarches n'empêche pas leur instrumentalisation et "l'agir éthique " peut simplement coïncider avec un simple souci, très libéral, d'accroître des performances financières. La vraie distinction à faire et à analyser serait peut-être entre "l'agir éthique " très en vogue, visible et lisible et un "être éthique ", révélateur de valeurs plus profondes qui guideraient un engagement de l'entreprise (Bossanne, 2006).

L'analyse que nous avons engagée montre comment des multinationales, des organisations non gouvernementales (ONG) ou des collectivités territoriales, ayant des objectifs divers (intentions mercantiles pour les premières, projet collectif pour les secondes) mobilisent les mêmes ressorts pour produire une communication sur le développement durable et l'environnement. Les projets sont différents, mais les moyens iconographiques ou les slogans font tous appel à la fois à l'émotionnel et à des référents de morale quasi religieuse. Ils mettent en exergue, non pas un processus de responsabilisation de la société dans son ensemble qui pourrait aboutir à réfléchir, construire, produire un projet collectif partagé, mais la culpabilité. Et c'est au regard de cette culpabilité, que ces multinationales, ONG ou collectivités territoriales nous proposent un discours "prêt à penser ", des méthodes "prêtes à appliquer » ou " prêtes à acheter ".

47 Faut-il alors prévoir une forme d'intervention publique destinée à contenir les excès du marketing écologique ? La réponse n'est pas simple tant les catégories de messages sont enchevêtrées les unes dans les autres. En outre, comment déplorer le marketing écologique de tel groupe multinational lorsque les organisations territoriales et politiques utilisent les mêmes ressorts pour gagner l'adhésion des citoyens ? De même, comment critiquer les simplifications de discours publicitaires vantant les mérites de la durabilité d'un produit ou sa qualité environnementale quand les associations écologistes brandissent elles aussi des slogans qui réduisent singulièrement la complexité des systèmes socio-environnementaux?

Le développement durable, en réconciliant les sphères de l'économique, de l'écologique et du social a abouti à donner au système économique une capacité à intégrer le discours de la doctrine, sans que finalement ne soit remis fondamentalement en cause le modèle de développement. Étrangement cependant, alors que « l'agir humain » et la notion de travail (ou d'emploi?) sont essentielles, une des trois dimensions du développement durable est absente dans l'ensemble du corpus documentaire que nous avons mobilisé : la dimension sociale. Comme si le registre du social disparaissait de l'intérêt collectif ou plutôt qu'il était induit aujourd'hui par l'économique. Ce prérequis idéologique (sans économie, point de social) s'impose aujourd'hui à l'ensemble des orientations politiques des États européens.

Le dernier titre de l'hebdomadaire Le Monde 2 est explicite à ce propos : «La nature va sauver l'industrie » ( $n^{\circ} 216$, Supplément au Monde $n^{\circ} 19658$ du 5 avril 2008). Dans un article du Point ( ${ }^{\circ} 1855$, du 3 avril 2008, p. 165) intitulé «Le Vert est dans le fruit", Frédéric Lewino nous propose de redescendre sur terre: «Ce tapage cache-t-il un grand bluff commercial ou les premiers signes d'une révolution verte? » questionne-til. "Pour en finir avec la pollution sans renoncer au développement, l'écologie industrielle propose de construire des villes et des usines qui s'inspirent de la nature ». Ainsi, la nature ne va pas nous permettre de mieux vivre, de mieux nous relier entrenous, d'y trouver des joies communes, mais seulement de pouvoir mieux faire fonctionner les entreprises et, à la rigueur, leurs employés. 
50 Le politique semble aussi faire les frais de cette évolution qui laisse aux forces économiques les leviers du projet de société : Total se joue ainsi des pouvoirs publics en affichant avec force, face à la formule obligatoire «l'énergie est notre avenir, économisons-la », son propre slogan qui ridiculise le premier « Pour vous notre énergie est inépuisable ». Véolia Transports fait peut-être mieux et plus subtil en s'attribuant, rien moins, les mérites des nouvelles politiques de la ville et du développement des transports en commun: "En transportant plus de 2,5 milliards de voyageurs chaque année, Véolia Transport libère l'espace urbain [...] » vante en effet une des publicités de la firme.

51 Ce sont enfin les instances éducatives qui sont touchées de plein fouet par cet écomarketing et cette prééminence des sphères de la communication sur celles de la connaissance. La mystification est totale, les éducateurs sont embarqués dans le tourbillon. Un manuel d'économie de terminale STG (sciences et technologies de la gestion) (Le Bolloch et al., 2006) présente ainsi aux élèves des publicités de Carrefour, Véolia et Gaz de France comme une manifestation de l'engagement effectif des industriels dans le projet de développement durable : "Véolia nous incite à exiger du progrès qu'il préserve la vie. Gaz de France nous assure qu'il travaille " pour demain ». Et Carrefour veut nous faire prendre conscience de l'urgence qu'il y a à "mieux consommer " " écrivent les auteurs du manuel en présentant des publicités de ces entreprises comme explication de ce qu'est le développement durable, à côté d'un extrait d'ouvrage de Michel Edouard Leclerc, président d'une grande chaîne d'hypermarchés.

La connaissance académique est court-circuitée à l'école même: le comité national «l'école agit » (le Grenelle de l'environnement à l'école) est porté par le ministère de l'Éducation nationale et hébergé sur le site du centre national de la documentation pédagogique ; c'est lui qui doit sélectionner des projets d'établissements exemplaires dans le domaine du développement durable. Cette instance est présidée par Nicolas Vanier, un de ces héros écolos de la sphère médiatique, et compte parmi ses membres éminents la présentatrice de la météorologie de TF1 Evelyne Dhéliat, entre un professeur d'université et un député de la République : ouverture de l'école à la société civile?

53 Au final, à travers l'ensemble de ces stratégies de communication, on aboutit à une conformation, un regard unique sur le développement durable. Cette conformation relève de deux compromissions. L'une plus individuelle et ciblée sur des personnages qui créent leur autorité scientifique dans la société à travers les médias, ce que Bourdieu appelait la complaisance à l'hétéronomie. Pour le développement durable, l'hétéronomie relève des " lois du commerce ", « de l'économie " qui sortent du champ spécifique de la science et de sa critique par l'expérimentation, la réflexion, l'incertitude, la précaution, etc. L'autre, plus collective, autour de la mise en place d'une "novlangue ", d'un nouveau langage apparemment partagé par tous et qui véhiculerait nos façons collectives d'être et de faire, ce que Eric Hazan (2006) à la suite des travaux du philologue Viktor Klemperer sur la LTI (Lingua Tertii Emperii) appelle la Lingua Quintae Respublicae (ou LQR) la langue de la cinquième République, celle du néolibéralisme qui vend et vante à travers «sa propagande du quotidien " et de la proximité, l'inventivité, l'innovation et la compétitivité. Les politiques de l'État suivent le mouvement, en France, la Délégation interministérielle à l'aménagement et la compétitivité des territoires a remplacé la Délégation à l'aménagement du territoire et 
à l'action régionale. L'action régionale est ainsi remplacée par la compétitivité des territoires. Pour Jean-Louis Borloo, ministre de l'Écologie, de l'Énergie, du Développement durable et de l'Aménagement du territoire: "Le développement durable n'est pas une mode passagère ou un argument marketing, mais le fondement même de la compétitivité de demain" (Le Point $n^{\circ} 1855,3$ avril 2008, p. 170). Mais qu'est-ce que la compétitivité, si ce n'est l'orientation de l'ensemble de notre démarche existentielle dans l'idée d'écraser l'autre au niveau économique, voire social et politique? Autant de glissements sémantiques qui expriment notre nouvelle manière d'habiter le monde. Et pour reprendre les termes d'Émile Cioran (1995), « on habite sa langue comme on habite le monde». L'éducation à l'environnement éthique doit critiquer ces fausses évidences (Taken-for-granted), ces naturalisations des processus sociaux et économiques actuels fournis par des penseurs à la sauvette et repris par l'intelligentsia politique et économique. Chaque citoyen est un faiseur de choix, de décisions, de procédures, c'est la base de l'idée de démocratie. Toutes les publicités semblent nous le rappeler, mais encore faut-il avoir accès à la prise d'information pour construire son opinion propre et non uniquement avoir accès à une information prédigérée qui mène à la conformation des esprits.

Enfin, cette capacité très performante des multinationales et des médias à s'emparer du développement durable et de l'environnement ne signe-t-elle pas, d'une certaine façon, la fin de l'engagement politique dans le développement durable et l'éducation à l'environnement et au développement durable? Véolia s'attribue les mérites du changement de mode de déplacement en ville, EDF construit des liens avec l'éducation nationale dans le domaine de l'éducation à l'environnement et au développement durable. Ce recul du politique est inquiétant. En donnant les clés du développement durable à l'entreprise et aux médias, le système déresponsabilise la société et le citoyen, l'investissement collectif et individuel dans le projet d'éducation civique ou d'EEDD (éducation à l'environnement pour un développement durable) et dans l'action collective. L'éducation constitue bien alors un enjeu et un défi majeurs dans l'espoir de construire des comportements éthiques et responsables. Les formes que prendra notamment l'EEDD seront peut-être déterminantes à l'heure d'un changement éventuel de modèle démocratique, voire de modèle de développement. Pour ce faire, l'éducation doit redonner toute sa place et son rôle à l'apprentissage de l'esprit critique et de la controverse, pour les jeunes et les moins jeunes.

Mais éduquer à la critique, c'est permettre une remise en cause des hiérarchies institutionnalisées, des autorités «naturelles ", mais népotiques et ouvrir la porte à la fragilisation du pouvoir en place. Et quel pouvoir accepterait aujourd'hui cette remise en cause?

\section{BIBLIOGRAPHIE}

ADEME et BVP. (2007). La publicité est-elle respectueuse du développement durable ? Angers : ADEME. 
Austin, J. [1962] (1991). Quand dire, c'est faire. Paris : Éditions du Seuil.

Blanc, N. (2008). Éthique et esthétique de l'environnement. EspacesTemps.net. Textuel, 31.01.2008. Consulté en avril 2009 sur : http://espacestemps.net/document4102.html.

Boserup, E. (1965). The conditions of agricultural growth. The economics agrarian change under population pressure. Londres : Allen and Unwin.

Bossane, E. (2006). L'éthique d'entreprise : quelle place pour l'environnement ? Chambéry : ENSAM (École nationale supérieure des arts et métiers), Concours 2006 Promotion de l'éthique professionnelle. Consulté en avril 2009 sur : http://www.cge.asso.fr/presse/ Unesco/mentions/ D-201780 \%20ENSAM \%20Chamberry \%20Emile \%20Bossanne.pdf

Bourdieu, P. (1996). Sur la télévision. Suivi de l'emprise du journalisme. Paris : Raisons d'Agir Éditions.

Cioran, E. (1995). CEuvres. Paris : Gallimard.

Comte-Sponville, A. (1991). Morale ou éthique. Lettre internationale, 13, 10-17.

Cosgrove, D. (1994). Contested global visions : One-World, Whole-Earth, and the Appolo-SpacePhotographs. Annals of the Association of American Geographers, 84(2), 270-294.

Dassié, V. (2002). Tempêtes sur Versailles. Chronique d'une émotion. Ethnologies comparées, 4. Consulté en ligne en avril 2009 sur : http://alor.univ-montp3.fr/cerce/r4/v.d.htm.

Debord, G. (1996). La société du spectacle, $4^{\mathrm{e}}$ édition. Paris : Gallimard.

Diamond, J. (2006). Effondrement. Comment les sociétés décident de leur disparition ou de leur survie. Paris : Gallimard.

Dolan, K. (1999). Ethics, Animals and Science. Oxford : Blackwell Science.

Dortier, J.-F. (2006). île de Pâques : la catastrophe a-t-elle vraiment eu lieu ? Sciences Humaines, 174. Consulté en ligne en avril 2009 sur : http://www.scienceshumaines.com/ ile-de-paques--lacatastrophe-a-t-elle-vraiment-eu-lieu-_fr_14450.html.

Duvoux, N. (2007). Grandeur et décadence des sociétés humaines : à propos de Jared Diamond. Consulté en ligne en avril 2009 sur : http://www.laviedesidees.fr/Grandeur-et-decadence-dessocietes.html.

Feenberg, A. (2004). Repenser la technique, vers une technologie démocratique. Paris : La Découverte.

Gervereau, L. (2004). Voir, comprendre, analyser les images, $4^{\mathrm{e}}$ édition. Paris : La Découverte.

Hazan, E. (2006). LQR : La propagande du quotidien. Paris : Éditions Raisons d'Agir.

Huxley, A. [1932] (1977). Le meilleur des mondes. Paris : Plon.

Joly, M. (1993). Introduction à l'analyse de l'image. Paris : Nathan Université.

Le Bolloch P., Le Fiblec, Y. et Mouline, M.-C. (2006). Manuel d'économie de Terminale STG. Paris : Éditions Bertrand-Lacoste.

Le Moigne, J.-L. [1977] (2004). La théorie du système général, théorie de la modélisation. Paris : Presses Universitaires de France.

Le Naire, O. (2007). Versailles, avant... après... et côté jardins. Express, 27 septembre 2007.

Meirieu, P. (2007). Qu'est-ce que la pédagogie ? Cours à l'Université Lyon 2 Semestre 2007-2008. Consulté en avril 2009 sur : http://www.meirieu.com/COURS/L3/coursL3.htm. 
Rossi, G. (2000). L'ingérence écologique. Environnement et développement rural du nord au sud. Paris : CNRS Éditions.

Sauvageot, A. (1987). Figures de la publicité, figures du monde. Paris : Presses Universitaires de France.

Sauvé, L. (2002). L'éducation relative à l'environnement : possibilités et contraintes. Connexion, $\operatorname{XXVII}(1 / 2), 1-4$.

Tiffen, M., Mortimore, M. et Gichuki, F. (1994). More people, less erosion : environmental recovery in Kenya. New Jersey : John Wiley \& Sons.

Von Bertalanffy, L. [1968] (2002). Théorie générale des systèmes. Paris : Dunod.

\section{NOTES}

1. L'ensemble du corpus documentaire peut être consulté sur: http://critiquesdepub. blogspot.com/.

2. Circulaire n 2007-077 du 29 mars 2007 de l'Éducation nationale relative à la « seconde phase de généralisation de l'éducation au développement durable ».

3. L'éthique de l'environnement, née dans le monde anglo-saxon, se divise en plusieurs courants plus ou moins radicaux, que nous ne détaillerons pas ici.

4. Là encore, on peut voir que l'objectivité du développement durable n'existe pas et qu'il y a une multitude de constructions sémantiques sur la question, ce que nous appelons une interobjectivation.

5. L'arrêté du 28 novembre 2006 a imposé la mention de ce slogan dans la publicité faite par une entreprise vendant de l'électricité, de la chaleur ou du froid, des combustibles solides, liquides ou gazeux, des carburants, ou par les services afférents à l'utilisation de ces énergies, à partir du $1^{\mathrm{er}}$ mars 2007.

6. Voir : http://blogs.lexpress.fr/ecologie/ces_heros_ecolos.html.

7. Rappelons que cette question du choix réfère implicitement pour EDF à l'ouverture des marchés européens et à la perte du monopole que cette entreprise publique avait sur le marché français. Cette perte du monopole du fait de la dérégulation oblige les entreprises à prendre les devants en termes d'image pour inscrire durablement une sorte de monopole psychique. C'est le cas également pour France Telecom par exemple.

8. Dans le Morvan, le sapin de Noël soustrait près de 1500 hectares du territoire à la biodiversité pour couvrir un quart de la consommation française d'arbres de Noël. L'arbre de Noël est un produit de l'activité agricole et non forestière. Outre son impact paysager, l'utilisation massive de produits phytosanitaires a un impact sur la qualité du milieu. Certaines de ces plantations, réalisées de façon opportuniste sont abandonnées avant d'être exploitées et se transforment en bosquets timbres-postes. La monospécificité des peuplements d'épicéas qui les composent et l'absence d'entretien dont ils sont l'objet dégradent alors leur état sanitaire, ils peuvent constituer des foyers de dissémination parasitaire en direction de peuplements forestiers sains voisins.

9. Dans un article de Libération du 3 avril 2008, le président du WWF France a cette réflexion de gestionnaire économique à propos de la relation de l'ONG (organisation non gouvernementale) avec les grandes entreprises donatrices de l'association (Total, par exemple) : « Il serait presque souhaitable qu'on ait un concurrent ». Cette formule démontre l'hégémonie économique de l'organisation et son inscription sur un véritable marché de la cause écologique. Elle tranche avec l'idéal de désintéressement attaché aux associations ou ONG en général. 


\section{RÉSUMÉS}

Dans l'univers de la communication, l'enjeu environnemental est aujourd'hui recyclé en «argument commercial» par les stratégies de positionnement des organisations qui sont le plus souvent pointées du doigt par les écologistes. Cette tendance traduit la capacité du système à se dépasser par ses propres limites et à se protéger de ses contradictions successives en les intégrant à son discours. Ce projet est l'occasion d'étendre la question du marketing écologique aux slogans et à la mobilisation iconographique. Ce point de départ amène à confronter éthique de l'environnement, éthique de la communication et in fine éthique de l'éducation. Ainsi, comment déplorer le marketing écologique de tel groupe multinational lorsque les organisations territoriales et politiques utilisent les mêmes ressorts pour gagner l'adhésion des citoyens? De même, comment critiquer les simplifications des discours publicitaires vantant les mérites de la durabilité d'un produit ou sa qualité environnementale quand les associations écologistes et parfois d'éducation à l'environnement brandissent elles aussi des slogans qui réduisent singulièrement la complexité des systèmes socio-environnementaux ? Ce texte tente de mettre en regard les discours argumentés qui ont pour vocation de faire autorité et les discours symboliques, via l'univers de la publicité, qui jouent tout à la fois de la rhétorique visuelle et textuelle pour construire les catégories et les références qui leur sont associées sur la problématique du développement durable.

Nowadays, ecological arguments are gaining in importance within marketing programs. This way, companies can catch people's attention and anticipate green reactions about their own environmental impacts. Studying a corpus of advertising messages, we show how ecological marketing spreads throughout the whole society. The phenomenon is of course effective in commercial advertising but also through non governmental organisations and local authorities. This text allows us to confront ethics of the environment, ethics of communication and in fine ethics of education.

\section{AUTEURS}

\section{VÉRONIQUE ANDRÉ-LAMAT}

Agrégée de géographie est enseignante-chercheure à l'UMR ADES CNRS / Université de Bordeaux, ses travaux de recherches portent sur la construction des représentations de la nature et l'analyse de modes de gestion de l'environnement, et notamment sur les modalités d'élaboration de l'action publique environnementale.

\section{LAURENT COUDERCHET}

Enseignant-chercheur en géographie à l'Université de Bordeaux et à l'UMR 5185 ADES, CNRS, ses travaux portent sur les paysages et la reconnaissance géomatique des états de la nature : diagnostics experts, diagnostics profanes partagés. Discours et politiques publiques de préservation de la biodiversité participent de ses centres d'intérêt.

\section{ANDRÉ-FRÉDÉRIC HOYAUX}

Enseignant-chercheur à l'UMR ADES CNRS / Université de Bordeaux, il travaille avec des méthodologies qualitatives d'entretiens multiples soit de réactivation, soit d'explicitation sur support discursif ou iconique auprès de divers types d'acteurs. Ces méthodes permettent de 
mettre en lumière la capacité justificatrice des actions humaines dans l'espace et des conceptions idéologiques de l'espace. 